\title{
The Center for Reproducible Science at the University of Zurich
}

\author{
Eva Furrer*, Leonhard Held
}

University of Zurich, Center for Reproducible Science

* correspondence: eva.furrer@uzh.ch

\section{How did it start?}

As statisticians, we have been working on reproducibility in our own research and teaching activities for a long time, expressed early on mainly by the choice of $\mathrm{R}$ [1] and its predecessor S-plus for statistical computations.

Starting in 2010 EF came to the University of Zurich and started to work in LH's group of biostatisticians, who paid close attention to reproducible statistical computations. LH had just introduced reproducibility requirements as one of the editors of the Biometrical Journal [2]. As Department Chair, he started to roll-out a literate programming approach for all original research and for most of the consulting work for researchers from the medical faculty of the University of Zurich. This approach combines the computational and narrative part of a research paper into one single document, resulting in a dynamic report of the research that has been done. We also decided to teach the approach to the students of our newly created Master Program in Biostatistics, for which EF was responsible. In consulting work and in teaching, the activities by the Department of Biostatistics do not stop at reproducible computing and dynamic reporting. We also provide our expertise on sound research design and appropriate analysis, and we teach our biostatistics students to become such experts as well, always with the goal of avoiding "poor medical research" [3]. But the effect of these efforts remained limited to our immediate circle of influence, whereas the problem was decidedly more widespread; see e.g. the Lancet series introduced by Macleod et al. [4]. Therefore, towards the end of 2017 we agreed "to do something about reproducibility more broadly at the University of Zurich", and the idea for the Center for Reproducible Science (CRS) was born.

\section{How was it established?}

We started by contacting a set of like-minded researchers across different faculties of the university with a rough plan of what we wanted to do. Everybody was enthusiastic and so we set out to propose an official center of competence of the University of Zurich.

To this end, the website of the university states: "Centers of Competence are interdisciplinary cross-faculty scholarly networks in which researchers and research groups of the University of Zurich coordinate their work according to strategic objectives. [...] Researchers who would like to establish a Center of Competence first contact the Office of the Vice
President Research (Division Research Development) to clarify the suitability of the project for this form of organization and to discuss the further procedure."

We were lucky to have a Vice President for Research who was extremely supportive of the idea and promised that he did not see any obstacles for the proposed center to be approved and to receive the initial funding that the university offers for competence centers. He advised us to get the support of the leadership of the key faculties, namely the Faculty of Medicine, of Science, of Arts and Social Sciences, and of Business, Economics and Informatics. With the required development plan and a draft of the regulations, we asked the deans or vice deans of those four faculties to support us as well. Except for some administrative hurdles, we did not meet resistance or opposition.

The Center was officially approved by the Executive Board of the University in June 2018, and so we organized a kick-off event in September. The goal of the event was to attract general interest in reproducibility and replicability through an attractive line of talks by international experts from various disciplines, who had written influential articles on reproducibility, replicability, meta research and scientific reform, or who lead similar initiatives at their own universities.

The financial support from the University and the faculties made it possible to hire a postdoctoral researcher dedicated to further support and extend the CRS activities. A large number of training and community events have since been organized for a diverse range of $\mathrm{PhD}$ programmes and other audiences across the university. The current flagship activities are the Good Research Practice two-day courses that are offered once per semester for all $\mathrm{PhD}$ students and Postdocs of the University of Zurich. Not surprisingly, dynamic reporting is one of the topics we introduce in these courses. Other topics of the course are scientific integrity, planning and design of studies, open science principles, study protocols and preregistration, good statistical practices, analysis plans, and reporting guidelines. Several dedicated "lab pitches" have been offered to address the increasing number of inquiries from different research groups. A regular ReproducibiliTea journal club has also been established, which has been moved to an online format since the start of the pandemic. 


\section{Who are the key partners?}

The CRS would not exist without the support of the Executive Board of the University and the leadership of the involved faculties.

Early on we assembled representatives of each faculty who would serve on the steering committee of the CRS. This group was instrumental in providing interdisciplinary knowledge and networking. Moreover, the CRS has more than 30 members from the four faculties, who participate with their entire research groups in the center, as well as more than 15 individual fellows, mostly early career researchers, who actively participate in the activities of the center. Our backbone of members and fellows helps us to recruit students for our courses, participants for our community events and, in some cases, to write grant proposals.

We also keep good contact with the service units of the University of Zurich, such as the Data Services and Open Access of the main library, the Open Science delegates, the Graduate Campus, the Office for Animal Welfare \& 3R, and the scientific IT service. We have common training activities, mutually invite each other as speakers for events, and refer researchers to the appropriate services for specific questions.

Even in the midst of a pandemic, we managed to have the CRS idea spill over to a nationwide network, the Swiss Reproducibility Network. We had a very good blueprint with the UK Reproducibility Network for the creation of this network and, again, the immediate support and enthusiasm from the leadership, this time the leadership of the Swiss National Science Foundation and the Swiss Academies of the Arts and Sciences. The collaboration with the like-minded researchers in the network and its international sister networks in Europe and beyond is very dynamic and rewarding, and we remain amazed by the enthusiasm we meet everywhere.

\section{What have been the difficulties in establishing and maintaining the center?}

The instrument of a competence center that the University of Zurich offers has been a very good starting point for us. However, competence centers are approved with the expectation that the initial funding is used to consolidate efforts and to seek for internal or external longer-term funding. With the topics "rigor", "transparency", and "reproducibility", this has proved to be difficult since they are general concerns and not a specific research direction for which researchers can find financial support. In addition, the heavy focus on training as opposed to research has also ruled out a large part of the national and international funding options. Therefore, finding funding took more patience than anticipated and a close partnership with the university's leadership who came to understand the fundamental importance of the center.

The second difficulty is to get the attention of as many researchers at the university as possible. Our events are frequently visited by the same set of persons and we keep preaching to the choir. We have received help with this problem from our service unit partners, who keep inviting us to speak at various occasions and we count on an accumulation effect. Other efforts to increase visibility include working closely with the Open Science delegates of the university, who made sure that rigor and reproducibility were not forgotten in the Open Science Policy or in the establishment of the Swiss Reproducibility Network.

\section{What were the keys to its success?}

The five main keys to the success of the CRS were: Financial, but also in principle, support of the university's leadership

Involvement of the appropriate set of persons and hiring motivated staff

Knowledge of the university structures, including personal connections

Focus on the training of ECRs

Our own enthusiasm and long hours

\section{Where and what will the CRS be in five years?}

The end of the first approval period is approaching in 2022 , and we are currently in the process of developing a long-term perspective for the CRS, potentially giving it a slightly different and more sustainable organizational form within the University. We are broadening the training efforts towards courses for bachelor and master students, some of them specialized in specific $\mathrm{PhD}$ programmes, and common training activities with SwissRN partners. Last but not least, we aim to widen the CRS research agenda with an increasing amount of research on research, which increasingly attracts more interest from stakeholders and funders.

Conflict of Interest: the authors declare no conflict of interest.

\section{References}

[1] R Core Team (2020). R: A language and environment for statistical computing. R Foundation for Statistical Computing, Vienna, Austria. URL https://www.R-project.org/.

[2] Hothorn T, Held L, Friede T. Biometrical journal and reproducible research. Biom J. 2009 Aug;51(4):553-5. doi: 10.1002/bimj.200900154.

[3] Altman D G. The scandal of poor medical research. BMJ 1994; 308:283 doi: 10.1136/bmj.308.6924.283

[4] Macleod, Malcolm R et al. Biomedical research: increasing value, reducing waste. The Lancet, Volume 383, Issue 9912, 101 104. doi: 10.1016/S0140-6736(13)62329-6. 